\title{
Experimental and Automated Analysis Techniques for High-resolution Electrical Mapping of Small Intestine Slow Wave Activity
}

\author{
Timothy R Angeli, ${ }^{1,2}$ Gregory $0^{\prime}$ Grady, $^{1,3}$ Niranchan Paskaranandavadivel, ${ }^{1}$ Jonathan C Erickson, ${ }^{4}$ Peng Du, ${ }^{1}$ \\ Andrew J Pullan, ${ }^{1,2,5}$ lan P Bissett ${ }^{3}$ and Leo K Cheng ${ }^{1,5 *}$ \\ ${ }^{1}$ Auckland Bioengineering Institute, The University of Auckland, Auckland, New Zealand; ${ }^{2}$ Riddet Institute, Palmerston North, New Zealand; \\ ${ }^{3}$ Department of Surgery, The University of Auckland, Auckland, New Zealand; ${ }^{4}$ Department of Physics and Engineering, Washington and Lee \\ University, Virginia, USA; and ${ }^{5}$ Department of Surgery, Vanderbilt University, Nashville, Tennessee, USA
}

\begin{abstract}
Background/Aims
Small intestine motility is governed by an electrical slow wave activity, and abnormal slow wave events have been associated with intestinal dysmotility. High-resolution (HR) techniques are necessary to analyze slow wave propagation, but progress has been limited by few available electrode options and laborious manual analysis. This study presents novel methods for in vivo $\mathrm{HR}$ mapping of small intestine slow wave activity.
\end{abstract}

\section{Methods}

Recordings were obtained from along the porcine small intestine using flexible printed circuit board arrays (256 electrodes; 4 $\mathrm{mm}$ spacing). Filtering options were compared, and analysis was automated through adaptations of the falling-edge variable-threshold (FEVT) algorithm and graphical visualization tools.

\section{Results}

A Savitzky-Golay filter was chosen with polynomial-order 9 and window size 1.7 seconds, which maintained $94 \%$ of slow wave amplitude, $57 \%$ of gradient and achieved a noise correction ratio of 0.083 . Optimized FEVT parameters achieved $87 \%$ sensitivity and $90 \%$ positive-predictive value. Automated activation mapping and animation successfully revealed slow wave propagation patterns, and frequency, velocity, and amplitude were calculated and compared at 5 locations along the intestine (16.4 $\pm 0.3 \mathrm{cpm}, 13.4 \pm 1.7 \mathrm{~mm} / \mathrm{sec}$, and $43 \pm 6 \mu \mathrm{V}$, respectively, in the proximal jejunum).

\section{Conclusions}

The methods developed and validated here will greatly assist small intestine HR mapping, and will enable experimental and translational work to evaluate small intestine motility in health and disease.

\section{(J Neurogastroenterol Motil 2013;19:179-191)}

\section{Key Words}

Electrophysiology; Gastrointestinal motility; Interstitial cells of Cajal; Signal processing

Received: November 5, 2012 Revised: January 16, 2013 Accepted: January 23, 2013

(c) This is an Open Access article distributed under the terms of the Creative Commons Attribution Non-Commercial License (http://creativecommons. org/licenses/by-nc/3.0) which permits unrestricted non-commercial use, distribution, and reproduction in any medium, provided the original work is properly cited.

*Correspondence: Leo K Cheng, PhD

Auckland Bioengineering Institute, Private Bag 92019, Auckland 1142, New Zealand

Tel: +64-9-373-7599, Fax: +64-9-367-7157, E-mail: I.cheng@auckland.ac.nz

Andrew J Pullan deceased, March 2012.

Financial support: This work and authors were supported in part by grants from the National Institute of Health (Grant No. R01 DK64775), the New Zealand Health Research Council, and the Riddet Institute. TRA was supported by the Riddet Institute Earle Food Research Scholarship and the Royal Society of New Zealand R.H.T Bates Postgraduate Scholarship.

Conflicts of interest: None.

Author contributions: Planning and design of studies were conducted by TRA, GO, AJP, IB and LKC. Collection of data was performed by TRA, GO, NP, PD and LKC. Data analysis was performed by TRA, GO, NP, JC and PD. Manuscript was drafted by TRA and GO. All authors (except AJP) revised and approved the final version of the manuscript. 


\section{Introduction}

A number of coordinated regulating factors contribute to governing the motility of the small intestine, including neural, hormonal and bioelectrical systems. One of these factors is an underlying rhythmic electrical slow wave event that is generated and propagated by a network of interstitial cells of Cajal (ICC). These slow waves coordinate the timing, frequency, and direction of intestinal contractions. ${ }^{1}$ However, the mechanisms contributing to normal and abnormal small intestine slow wave pacesetting and propagation remain unclear and controversial, largely due to limitations of previous recording and analysis techniques.

Previous studies have measured slow wave activity in the small intestine, but most have been restricted to sparse recordings where a few electrodes are spaced along the intestine. ${ }^{2,3}$ Such methods provide information about slow wave morphology and frequency, but they are unable to provide spatial information about slow wave propagation dynamics, such as pacemaker activity and wavefront interactions. ${ }^{2}$ High-resolution (HR) techniques have more recently been introduced, whereby recordings are obtained from dense arrays of many electrodes, allowing activation sequences to be recorded in fine spatiotemporal detail. ${ }^{4-6}$ These HR mapping techniques have now been applied to evaluate gastric slow wave patterns in health and states of dysrhythmia, including in human motility disorders. ${ }^{7,8}$ Clinical interest in evaluating intestinal slow wave patterns has been fuelled by associations between a range of motility disorders and aberrant slow wave activity or ICC function, such as diabetic dysmotility, ${ }^{9}$ irritable bowel syndrome, ${ }^{10}$ and post-surgical dysmotility. ${ }^{11}$ However, there remains a critical lack of clinical intestinal slow wave studies to date.

While an automated pipeline of sophisticated algorithms now exists to support gastric mapping, ${ }^{12-14}$ there is no equivalent system for analyzing the vast data sets that result from HR small intestine mapping. This data throughput problem has been a major impediment to experimental and translational progress. In addition, small intestine HR mapping to date has been limited to flat, rigid electrode platforms. ${ }^{5,6}$ While these arrays achieve a high signal quality, they cannot conform to the intestinal curvature, so they can only be applied to a portion of the circumference. ${ }^{6,15}$

The aims of this study were therefore: (1) to develop reliable HR recording methods for in vivo intestinal electrical activity using flexible printed circuit board (PCB) arrays and (2) to improve the efficiency of intestinal slow wave data analysis through the development of automated algorithms. The practical utility of these advances was validated in a porcine model. This work builds on that initially presented in abstract form at the IEEE EMBS conference. ${ }^{16}$ In particular, the effects of 4 different filtering methods were quantified, intestine slow wave velocity and amplitude maps are presented, and a more complete set of experimental data was included in the optimization of the analysis algorithm parameters.

\section{Materials and Methods}

\section{Electrode Design and Application}

Flexible PCB arrays were designed for intestinal recordings, based on those validated for gastric mapping. ${ }^{4}$ These arrays consisted of gold contacts with copper connectors, embedded in a polyimide base. The recording head of each PCB consisted of 32 electrodes $(0.3 \mathrm{~mm}$ diameter) arranged in a $16 \times 2$ array, with an inter-electrode spacing of $4 \mathrm{~mm}$. The $4 \mathrm{~mm}$ spacing was calculated to be sufficient for recording small intestine slow waves based on the spatial variant of the Nyquist theorem. ${ }^{17}$ Eight PCBs were used simultaneously in each recording, comprising 256 electrodes in a $32 \times 8$ array, covering $124 \mathrm{~mm}$ of intestinal length and approximately $35 \mathrm{~cm}^{2}$ (Fig. 1A). The PCB arrays were contained in gauze-padded silicone cradles that were sized to match the circumference of the porcine jejunum (Figs. 1B and 1C). The cradled arrays were placed such that the electrodes conformed to the intestinal curvature, covering most of the circumference, while exerting only gentle pressure on the serosa (Fig. 1D). Small windows were made in the mesenteric attachments at the edges of the cradles, through which gauze was tied over the top of the cradles to gently hold the electrodes in contact.

\section{Experimental Recordings}

Ethical approval for this work was obtained from the University of Auckland Animal Ethics Committee (R698). All experiments were performed in vivo on white, cross-breed, weaner pigs of either sex $(\mathrm{n}=5 ; 34.9 \pm 0.7 \mathrm{~kg})$. Animal preparation and anesthesia were as previously described in another study evaluating gastric slow wave activity. ${ }^{18}$ In brief, the animals were fasted overnight and then subjected to general anesthesia, which was induced with Zoletil (Tiletamine $\mathrm{HCl} 50 \mathrm{mg} / \mathrm{mL}$ and Zolazepam $\mathrm{HCl} 50 \mathrm{mg} / \mathrm{mL}$ ) and maintained with isoflurane (2.5-5\% with an oxygen flow of $400 \mathrm{~mL}$ within a closed circuit anesthetic system). Slow wave activity has been successfully recorded under anes- 

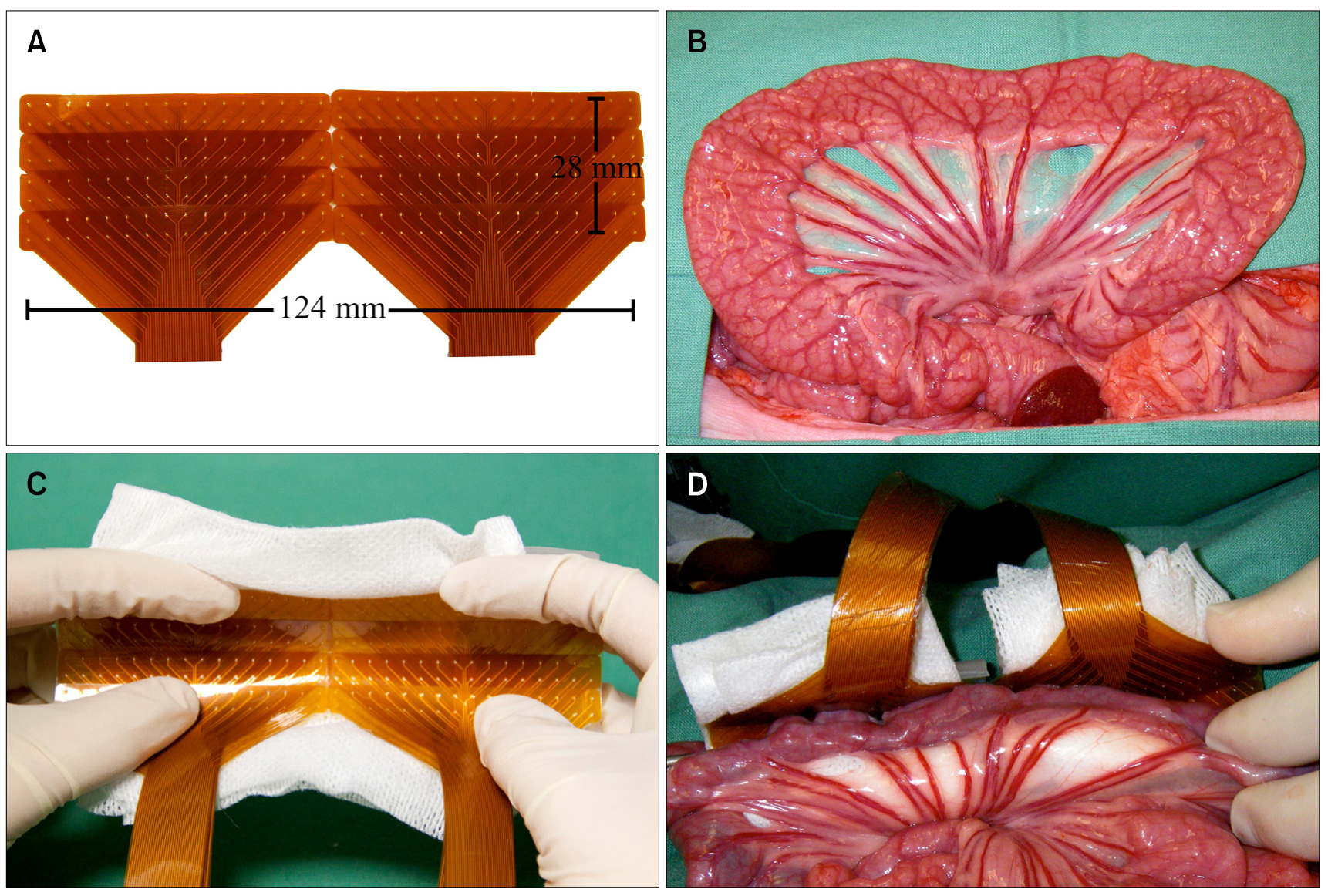

Figure 1. In vivo application of electrodes. (A) Eight printed circuit board (PCB) electrode arrays were tessellated to create a large rectangular electrode array with 256 electrodes (arranged in 8 by 32 configuration with $4 \mathrm{~mm}$ inter-electrode spacing). (B) Exteriorized small bowel segment. (C) Electrode array layered in gauze padded silicon cradles. (D) Small bowel segment held in contact with the electrode array by the silicon cradle.

thetic in many previous studies in several species, ${ }^{6,8,18}$ and anesthesia of this type is thought not to have a major effect on slow wave activity.

Following midline laparotomy, the flexible PCBs were applied to the serosa at intervals along the small intestine. The porcine small intestine was approximately $15.5 \mathrm{~m}$ in length, and the first recording was obtained from the duodenum, immediately distal to the pylorus. Two jejunal recordings were taken, the first immediately distal to the ligament of Treitz (approximately $0.5 \mathrm{~m}$ distal to the pylorus in pigs; proximal jejunum) and the second at $4 \mathrm{~m}$ distal to the ligament of Treitz (mid-jejunum). Two ileal recordings were then obtained, the first was at 9 meters distal to the ligament of Treitz (proximal ileum) and the second was at $10 \mathrm{~cm}$ proximal to the ileo-cecal junction (distal ileum). Visceral handing was kept to a minimum. The intestine was placed back into the abdomen during recordings, and the wound edges were approximated and packed with warm wet gauze. Unipolar record- ings were acquired at a sampling frequency of $512 \mathrm{~Hz}$ using an ActiveTwo System (Biosemi, Amsterdam, The Netherlands), with reference electrodes on the hind leg.

\section{Filtering}

Raw data was down-sampled to $30 \mathrm{~Hz}$ for computational efficiency, and baseline wander was removed using a Gaussian moving median filter with 20 second moving window. ${ }^{19}$ The frequency of interest was determined to be in the $0-120 \mathrm{cpm}(0-2$ $\mathrm{Hz}$ ) range by analyzing the frequency domain via a Fourier transform of the baseline-removed signal (Figs. 2A and 2B). The performances of 4 digital filters were investigated, with parameters set to accommodate the frequency of interest and as previously set for gastrointestinal extracellular studies: SavitzkyGolay filter (polynomial-order 9, window size 1.7 seconds), Butterworth band-pass filter (5-60 cpm), Butterworth low-pass filter $(120 \mathrm{cpm})$, and continuous wavelet transform (CWT) 
A

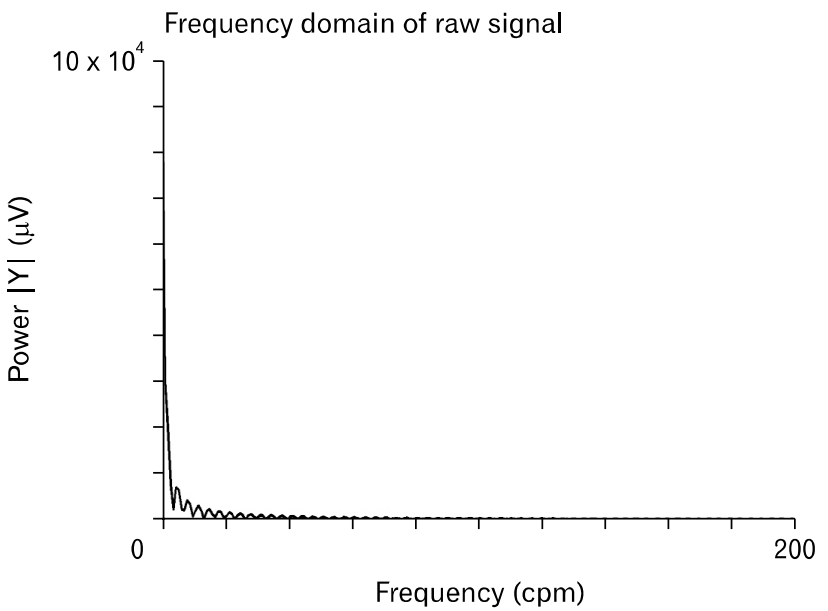

C

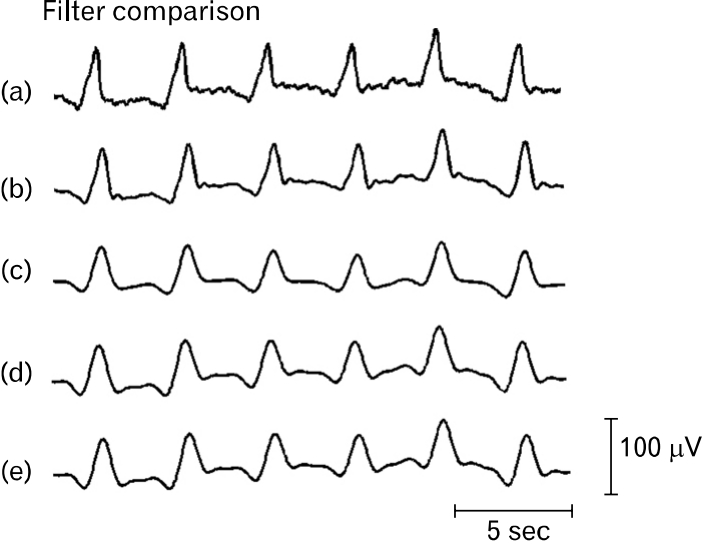

band-pass filter $(5-60 \mathrm{cpm}) .^{19}$

Slow wave activation times (ATs) were manually marked in 30 unfiltered channels from 3 separate recordings ( $\mathrm{n}=3$ pigs). Manual marking was performed by visually determining the point of steepest negative down-slope on the slow wave, which corresponds with the arrival of the wavefront under the recording electrode. ${ }^{12}$ Using these manually-marked ATs, 3 metrics were computed for evaluating the filters: noise correction ratio (NCR), slow wave amplitude and slow wave gradient. NCR estimated the level of noise removed in sections of the iso-electric regions of the leads, computed as,

$$
\mathrm{NCR}=\frac{\left\|\mathrm{n}_{\text {unfit }}-\mathrm{n}_{\text {fitt }}\right\|_{2}}{\left\|\mathrm{n}_{\text {unfit }}\right\|_{2}}
$$

where $\mathrm{n}_{\text {unfit }}$ is the noise of the iso-electric regions of the unfiltered signal, $\mathrm{n}_{\text {filt }}$ is the noise of the iso-electric regions of the filtered signal, both after baseline removal, and $\|. . .\|_{2}$ is the Euclidean norm.
B

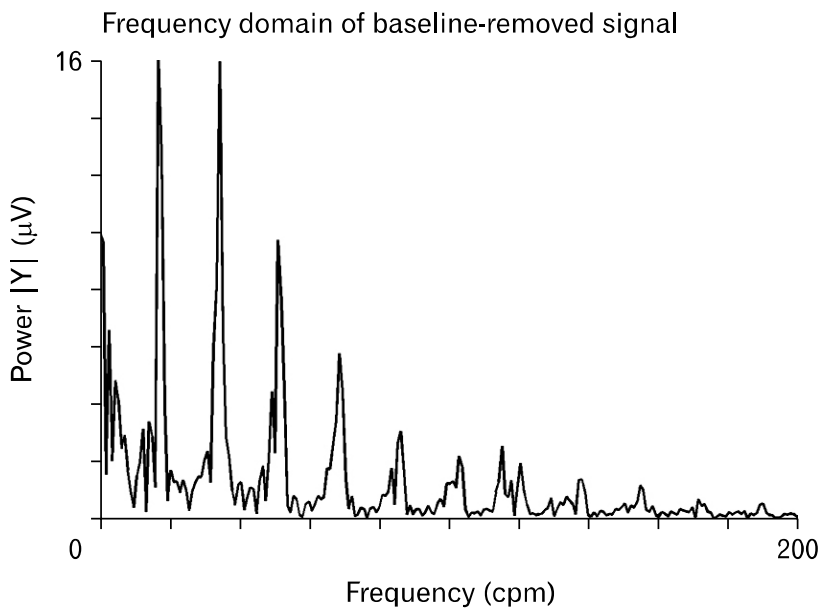

Figure 2. Filtering of intestinal slow waves. (A) The frequency domain of the raw unfiltered signal shows high levels of low-frequency noise. (B) The frequency domain of the baseline-removed signal shows that the moving median filter effectively removes the low-frequency noise and the frequency of interest lies in the range of $0-120 \mathrm{cpm}(0-2$ $\mathrm{Hz}$ ). (C) A representative raw slow wave signal from one channel of the printed circuit board electrode (a) is compared to the filtered form using the 4 selected filters: Savitzky-Golay (b); Butterworth 5-60 cpm (c); Butterworth $120 \mathrm{cpm}$ (d); and CWT 5-60 cpm (e). The SavitzkyGolay filter maintained the best signal quality (see also Fig. 4).

Amplitude was computed by placing a window of 10 sample points (i.e., one-third of a second) on either side of the AT, and taking the difference of the maximum and minimum values. The gradient of the slow wave down-stroke was computed using a 3 point central difference. NCR, amplitude, and gradient were compared between the filtered and unfiltered signals, to determine the optimal filter for removing low and high frequency noise while maintaining signal integrity.

\section{Signal Analysis}

Data analysis was performed in the Gastrointestinal Electrical Mapping Suite (GEMS) v1.5. ${ }^{20}$ An automated marking algorithm (falling-edge variable-threshold $[\mathrm{FEVT}]$ ) was adapted and tuned for marking the small intestine ATs. ${ }^{12}$ In brief, FEVT calculates a specified signal transform and convolves the transformed signal and a falling-edge kernel. It then utilizes a time-varying threshold for detection of times when the convolved 
signals exceed the threshold, thereby identifying ATs at the point of steepest negative descent. The FEVT algorithm includes several tunable parameters, including a threshold multiplier $(\eta)$, refractory period $\left(T_{r}\right)$, smoothing kernel width $(\rho)$, and running median window half-width $\left(\tau_{\mathrm{HW}}\right)$. The $\eta$ defines the threshold which the signal must surpass in order to be considered as a slow wave event, $T_{r}$ defines the minimum period between FEVT marks, $\rho$ defines the time width of the moving average filter applied to the detection signal transform, and $\tau_{H W}$ defines the time window for which the time-varying threshold is computed. ${ }^{12}$ Additionally, the signal transform used for the FEVT algorithm was also optimized between 4 different methods: negative derivative, amplitude-sensitive differentiator, non-linear energy op-
Table 1. Falling Edge Variable Threshold Algorithm Parameter Tuning

\begin{tabular}{llc}
\hline \multicolumn{1}{c}{ Parameter } & \multicolumn{1}{c}{ Values tested } & Optimal value \\
\hline Signal transform & ND, ASD, NEO, DEO4 & NEO \\
$\eta$ (unit-less) & $2,3,4$ & 2 \\
$T_{\mathrm{r}}(\mathrm{sec})$ & $0.5,1,1.5,2,2.5$ & 2 \\
$\rho(\mathrm{sec})$ & $0,1,2$ & 0 \\
$\tau_{\mathrm{HW}}(\mathrm{sec})$ & $10,15,20$ & 10 \\
\hline
\end{tabular}

A range of values of the FEVT signal transform were systematically combined and tested as listed in column 2. The parameter values that resulted in the combined best performance across all 3 data sets are shown in the third column and yielded performance metrics as presented in Table 2 .

$\mathrm{ND}$, negative derivative; ASD, amplitude-sensitive differentiator; NEO, non-linear energy operator; DEO4, fourth-order differential energy operator; FEVT, falling edge variable threshold.
A

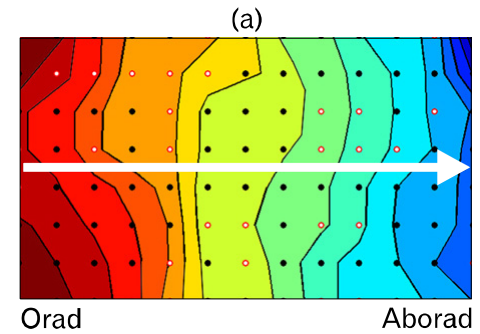

B

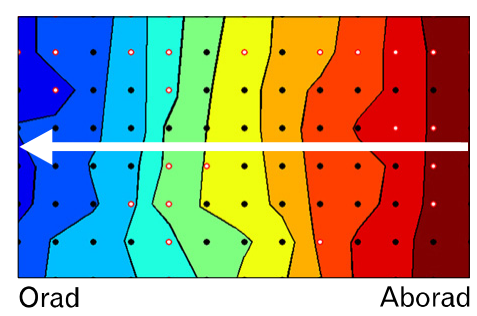

C

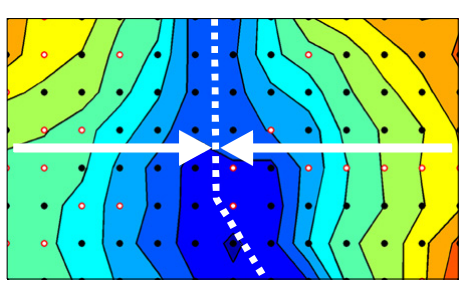

Orad

Aborad
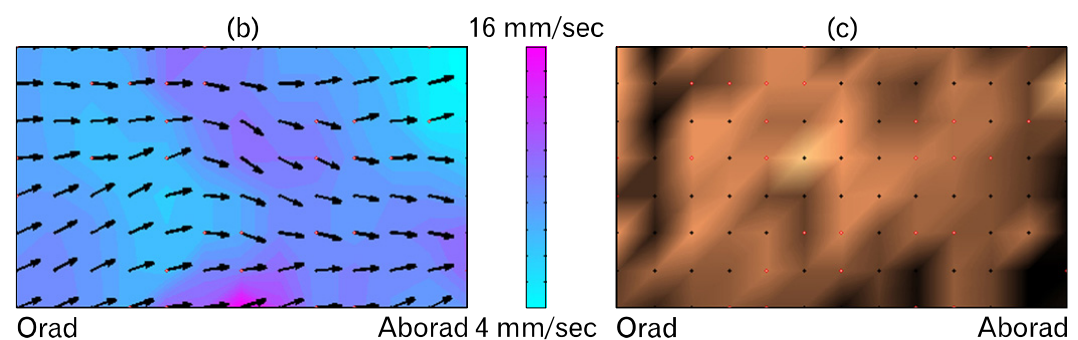

$63 \mu \mathrm{V}$

Orad

Aborad $4 \mathrm{~mm} / \mathrm{sec}$ Orad

Aborad $15 \mu \mathrm{V}$

$10 \mathrm{~mm} / \mathrm{sec}$
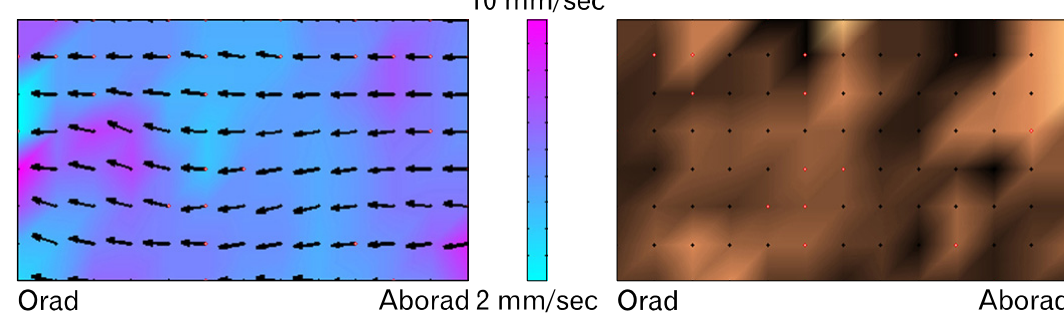

$78 \mu \mathrm{V}$

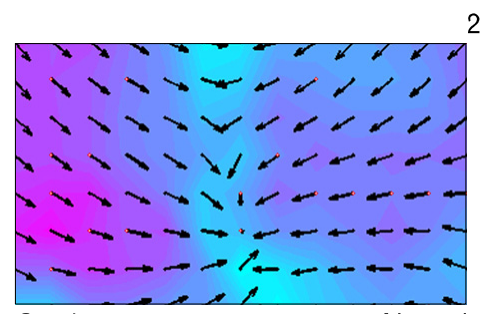

$24 \mathrm{~mm} / \mathrm{sec}$
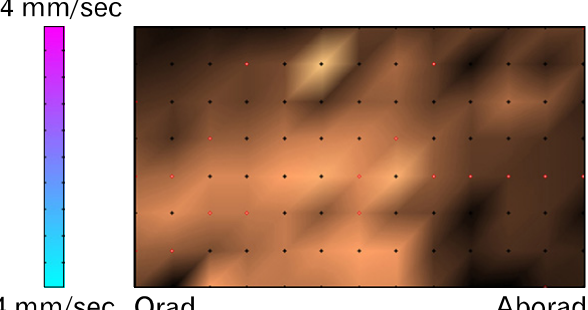

$51 \mu \mathrm{V}$

Orad

Aborad $4 \mathrm{~mm} / \mathrm{sec}$ Orad

Aborad $15 \mu \mathrm{V}$

Figure 3. Isochronal activation time (a), velocity field (b) and amplitude (c) maps of small intestine slow wave activity. The electrode array was wrapped around the circumference of the jejunum such that the top and bottom of each map correspond to nearly-adjacent tissue at the mesenteric border. Three propagation examples are presented: (A) Consistent antegrade propagation (isochronal interval 0.5 seconds; see also Video 1). (B) Consistent retrograde propagation (isochronal spacing $=1.0$ second; see also Video 2). (C) Colliding slow wave fronts. Slow wave activity originates both oral and aboral to the array and collides in the middle (dashed line); (isochronal spacing $=0.25$ seconds; see also Video 3 ). The activation maps (a) show a single wave front, with each color band indicating the area of slow wave propagation per unit time, progressing from red (early) to blue (late). The velocity field maps (b) show slow wave propagation direction at each electrode with an arrow, and the magnitude of velocity is displayed as a color gradient, according to the scale shown. The amplitude maps (c) display the slow wave amplitude as a color gradient according to the scale shown. Each black dot represents an electrode, and white dots outlined in red represent electrodes where activity was interpolated. 
erator, and fourth-order differential energy operator. ${ }^{12}$ The negative derivative method computes the first-derivative of the signal and considers only those values which correspond to a negative deflection in the signal. The latter 3 of these signal transforms can essentially be categorized as the first, second and fourth order differential energy operators. The transformed signal accentuates high-amplitude, high-frequency components of the original recordings. In general, the nth order energy transform is proportional to $A_{n} \omega_{n}$, where $A$ and $\omega$ are the instantaneous amplitude and frequency of the original signal, respectively. ${ }^{21}$

FEVT parameter values and signal transforms were incrementally combined to analyze 3 representative data segments from the porcine jejunum ( $\mathrm{n}=3$ pigs), with values as shown in Table 1. FEVT results were compared to the manually-marked data sets to determine true positive (TP), false positive (FP) and false negative (FN) AT results. Hand-marked data were independently verified by 3 experienced markers to ensure accuracy, and only marks that all 3 markers agreed upon were used. A TP result corresponded to the FEVT mark placed within one second of a hand-marked event, a FP result corresponded to an erroneously placed FEVT mark, and a FN result corresponded to a falsely omitted FEVT mark. Three performance metrics were calculated based on the TP, FP and FN results, as follows:

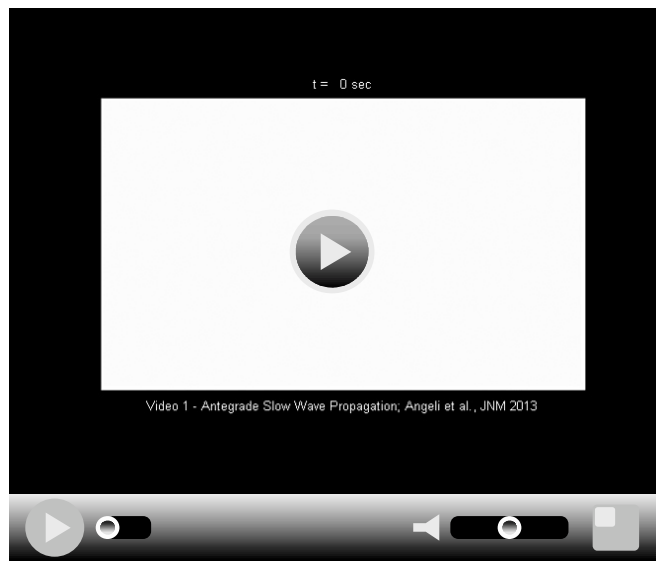

Video 1. Slow wave propagation in the antegrade direction. Each pixel represents an electrode and lights up when the slow wave passes that electrode. Slow wave activity propagates from orad (left) to aborad (right), and sequential slow waves are colored differently for easy differentiation. (see also Fig. 3A) (Video clip is available at http://www. jnmjournal.org/journal/journal_view.html?year $=2013 \& \mathrm{vol}=019 \& \mathrm{num}$ $=02 \&$ page $=179)$.
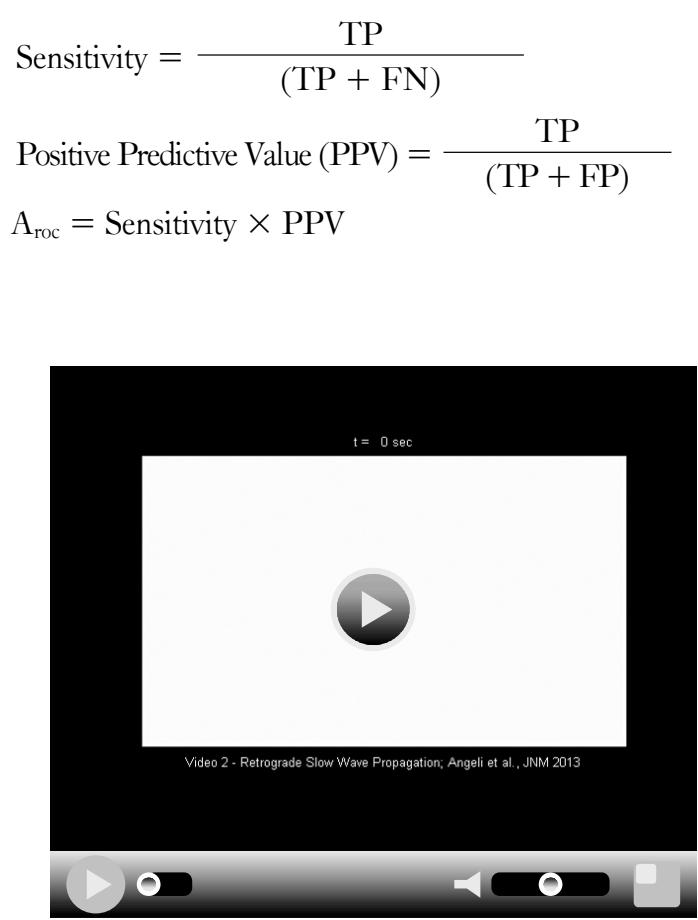

Video 2. Slow wave propagation in the retrograde direction. Each pixel represents an electrode and lights up when the slow wave passes that electrode. Slow wave activity propagates from aborad (right) to orad (left), and sequential slow waves are colored differently for easy differentiation (see also Fig. 3B) (Video clip is available at http://www. jnmjournal.org/journal/journal_view.html?year $=2013 \& \mathrm{vol}=019 \&$ num $=02$ \&page $=179$ ).

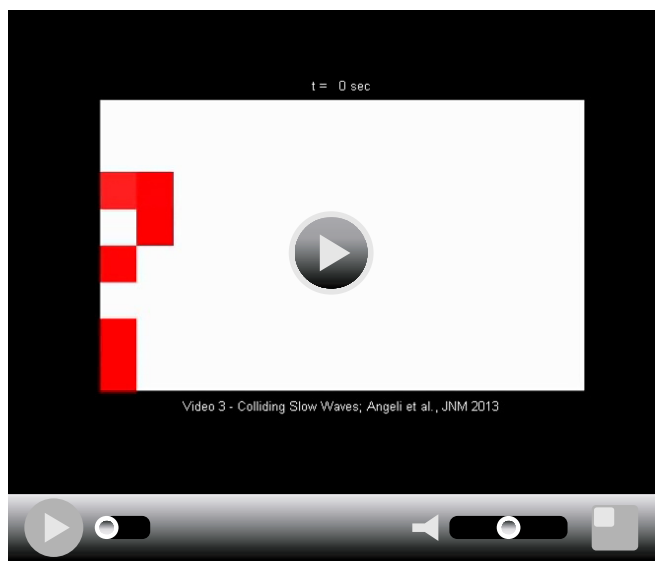

Video 3. Colliding slow wave activity. Each pixel represents an electrode and lights up when a slow wave passes that electrode. This recording shows 2 slow wave fronts, one travelling antegrade and the other retrograde, colliding and terminating in the middle of the animation frame (see also Fig. 3C). Separate slow wave fronts are colored differently for easy differentiation. (Video clip is available at http://www. jnmjournal.org/journal/journal_view.html?year $=2013 \& v o l=019 \&$ num $=02 \&$ page $=179$ ). 


\section{Visualization of Slow Wave Activity}

Experimental data analysis was performed to show experimental validation and proof of utility on 24 total recordings, encompassing greater than 11,000 individual slow wave ATs, from 5 locations along the intestine ( $\mathrm{n}=6$ pigs): proximal duodenum, proximal jejunum, mid-jejunum, proximal ileum and terminal ileum. All automatically detected ATs were manually reviewed to ensure accuracy. An automated grouping algorithm ('REGROUPS') was applied to cluster individual slow waves into coherent wavefronts, ${ }^{13}$ and manually reviewed. Automated isochronal mapping of ATs was then performed to display and quantify the propagation of successive wavefronts in isochronal time maps, ${ }^{13}$ as shown in Figure 3. Animations were also generated to visualize slow wave propagation as flashing pixels, representing ATs occurring at each electrode, as shown in Videos $1-3$.
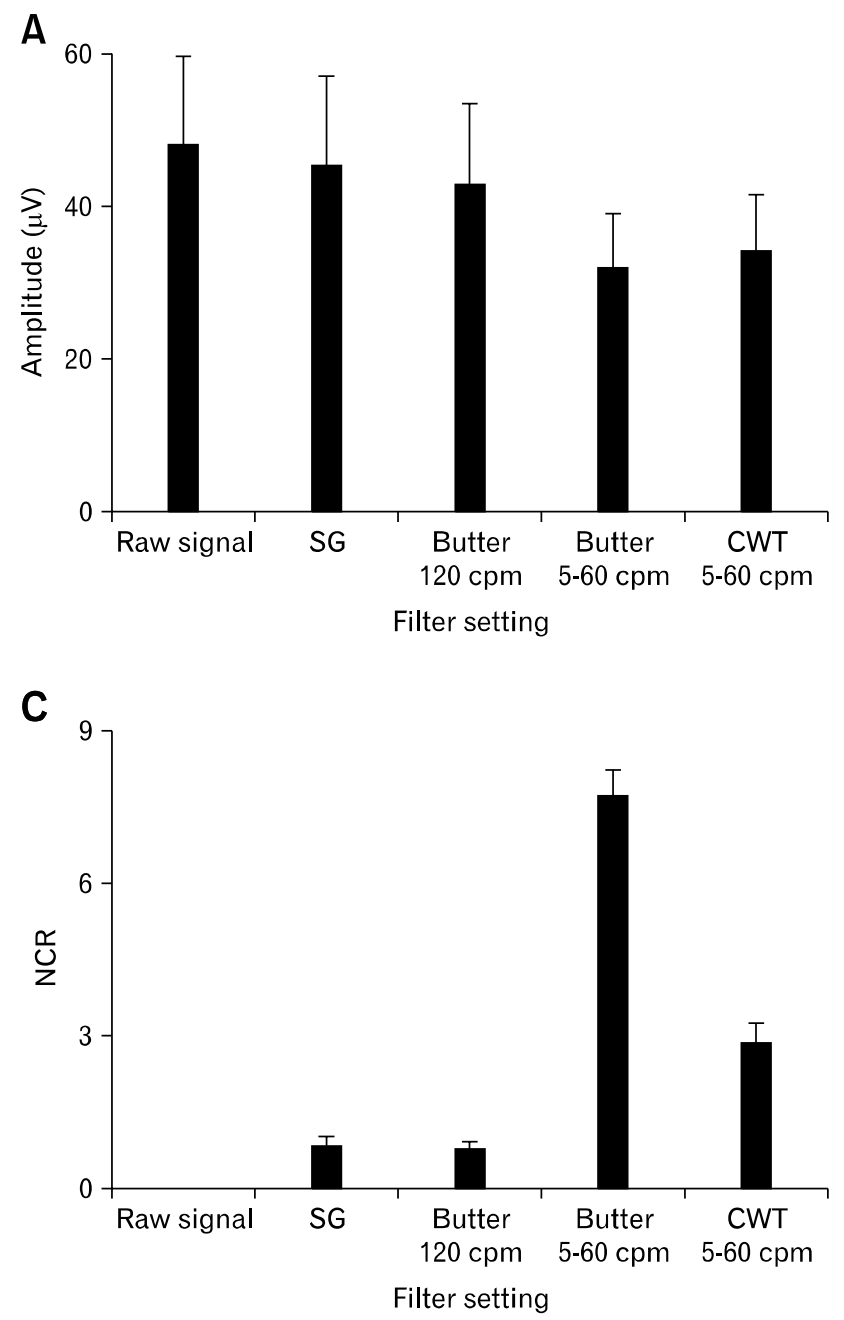

\section{Analysis of Slow Wave Characteristics}

Slow wave frequency, amplitude and velocity were calculated on the same 24 recordings described above. Frequency was calculated by determining the time between subsequent slow waves and is reported in cycles per minute (cpm). The amplitude of each slow wave was determined by detecting the peak and trough using a zero-crossing approach within a 1.5 seconds window centered at the AT, and the velocity was calculated by using a validated smoothed finite difference approach. ${ }^{14,19}$ Frequency, amplitude and velocity were compared between antegrade and retrograde propagating slow waves. Automated velocity field and amplitude maps were generated to visualize these characteristics in spatial detail (Fig. 3). Frequency, amplitude, and velocity are reported as mean values with standard deviation (SD) or standard error of the mean (SEM).

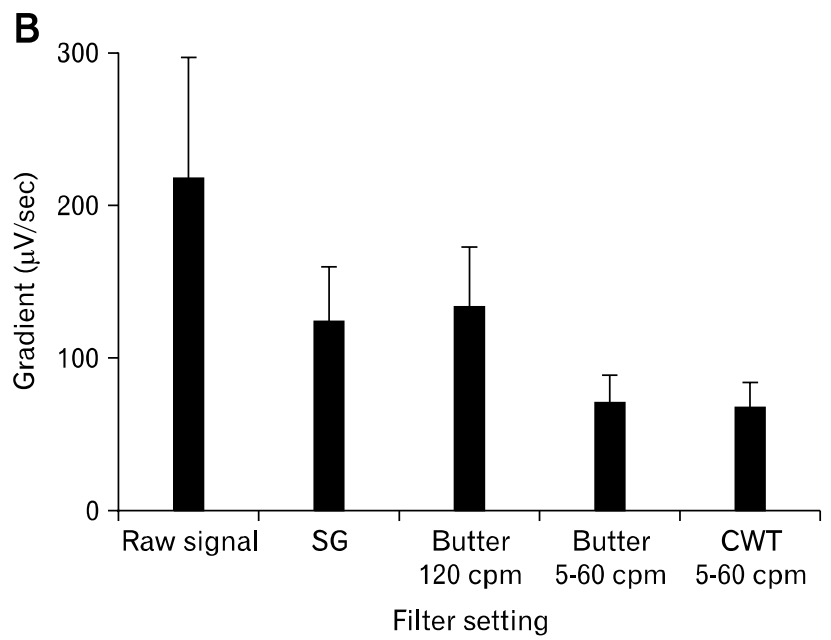

Figure 4. Signal comparison of 4 filters previously used for gastrointestinal extracellular recordings. Slow wave amplitude (A), slow wave gradient $(\mathrm{B})$ and noise correction ratio $(\mathrm{NCR})(\mathrm{C})$ were compared. The Savitzky-Golay (SG) filter with polynomial-order 9 and window size 1.7 seconds performed very similar to the low-pass Butterworth filter of 120 cpm, out-performing the band-pass Butterworth filter of 5-60 cpm and the continuous wavelet transform filter of $5-60 \mathrm{cpm}$ in regards to maintaining slow wave amplitude and gradient. The SG filter proved to be the best filter for small intestine slow waves. The Butterworth filter of 5-60 cpm achieved the best NCR, and may prove useful for recordings that have large amounts of noise interference, but it must be noted that the slow wave amplitude and gradient are appreciably decreased with this filter. Butter, Butterworth; CWT, continuous wavelet transform. 


\section{Results}

\section{Experimental Recording}

The flexible PCBs were successfully utilized to record in vivo small intestine slow wave activity, yielding readily identifiable slow wave events, as shown in Figure 2C. They covered a length 2.5 times greater than previous studies (124 versus $46 \mathrm{~mm}$ ). ${ }^{6,22}$ The silicone cradles effectively conformed the flexible PCBs around the intestinal circumference and held the electrodes in contact with the serosa, yielding slow wave recordings in a mean of $75 \pm 9 \%$ of the channels.

\section{Signal Processing}

The Savitzky-Golay filter with polynomial-order 9 and window size 1.7 seconds best maintained the slow wave amplitude, as shown in Figures 2 and 4. This filter maintained 94\% of the amplitude of the raw signal, compared to $89 \%$ with the Butterworth $120 \mathrm{cpm}$ filter, $66 \%$ with the Butterworth 5-60 cpm filter and $71 \%$ with the CWT filter. The down-slope was more significantly affected by all filtering methods because these filters are designed to remove high-frequency content and smooth the signal, but the Savitzky-Golay filter once again performed well, although slightly below the Butterworth $120 \mathrm{cpm}$ filter. The Savitzky-Golay filter maintained a gradient of $57 \%$ that of the raw signal, compared to $61 \%$ with the Butterworth $120 \mathrm{cpm}$ filter, $33 \%$ with the Butterworth 5-60 cpm filter, and $31 \%$ with the CWT filter. Although the Butterworth filter of 5-60 cpm performed poorly in maintaining the slow wave amplitude and down-slope, it vastly outperformed the other filters in noise cor-

Table 2. Performance Metrics of Tuned Falling Edge Variable Threshold Algorithm

\begin{tabular}{lc}
\hline Performance metric & $\begin{array}{c}\text { Value after tuning of FEVT } \\
\text { parameters (mean } \pm \text { SD) }\end{array}$ \\
\hline A roc $_{\text {Sensitivity }}$ & $0.79 \pm 0.08$ \\
PPV & $0.87 \pm 0.04$ \\
TP rate & $0.90 \pm 0.07$ \\
FN rate & $0.87 \pm 0.04$ \\
FP rate & $0.13 \pm 0.04$ \\
\hline
\end{tabular}

Average falling edge variable threshold (FEVT) performance over the 3 validation data sets using the optimal FEVT parameters presented in Table 1. Results exclude channels lacking slow wave signal content.

Aroc, area under the receiver operating characteristic curve; PPV, positive predictive value; $\mathrm{TP}$, true positive; $\mathrm{FN}$, false negative; $\mathrm{FP}$, false positive. rection, with an NCR of 0.78 , followed by the CWT filter at 0.29, the Savitzky-Golay filter at 0.083, and the Butterworth 120 cpm filter at 0.078 .

\section{Automated Analysis Algorithm Optimization}

The FEVT algorithm parameters were optimized and validated against a total of 7,525 manually-marked and validated slow wave ATs over 3 data sets ( $n=3$ pigs). Since sensitivity and positive predictive value (PPV) are inversely correlated in practice, ${ }^{12}$ area under the receiver operating characteristic curve $\left(A_{\text {roc }}\right)$ served as the best overall measure of FEVT performance. A comparison of the $A_{\text {roc }}$ values for each combination of FEVT parameter values across all 3 data sets yielded the optimal set of parameter values of: non-linear energy operator signal transform, $\eta=3$, $\mathrm{T}_{\mathrm{r}}=2$ seconds, $\rho=0$ seconds and $\tau_{\mathrm{HW}}=10$ seconds (summarized in Table 1).

Channels that did not contain any slow wave signals, likely due to poor contact between the electrode and intestinal serosa, were removed from analysis. The performance of the FEVT algorithm with the above optimal parameter settings demonstrated an average PPV of 0.90 and Sensitivity of 0.87 , which yielded an average $A_{\text {roc }}$ value of 0.79 . Additional performance metrics are summarized in Table 2 and illustrated in Figure 5.

\section{Visualization of Slow Wave Propagation}

Activation maps and animations were constructed to visualize the propagation patterns of intestinal slow waves and show proof of utility of the developed methods. The results of the

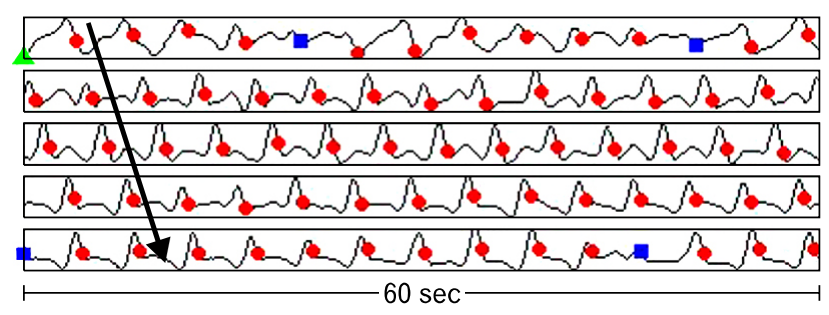

Figure 5. Five electrograms of slow wave activity from the porcine jejunum with automated slow wave identification by the falling edge variable threshold (FEVT) algorithm represented as colored marks. Red circles represent a true positive mark, green triangles represent a false positive mark, and blue squares represent a false negative mark. Amplitude is automatically scaled for each individual channel, but averaged $32 \pm 6 \mu \mathrm{V}$ in the mid-jejunum. The 5 electrograms shown here are from adjacent electrodes, such that slow wave propagation across the channels can be seen, as represented by the arrow. After tuning of the FEVT parameters across 3 data sets from 3 pigs, the algorithm achieved $90 \%$ positive-predictive value and $87 \%$ sensitivity. 


\section{Average slow wave characteristics}
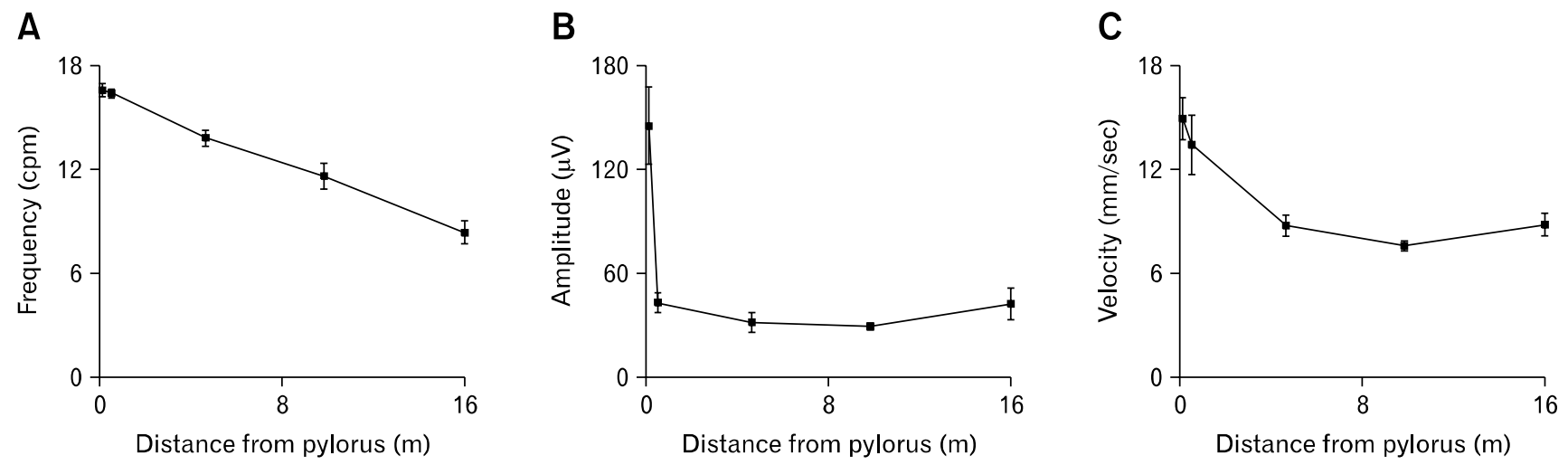

Figure 6. Average slow wave characteristics from five locations along the small intestine: proximal duodenum, proximal jejunum, mid-jejunum, proximal ileum and terminal ileum. Frequency (A), amplitude (B) and velocity (C) all decreased along the intestine. However, the rates of decrease of each were different, as illustrated in the graphs. Quantitative values are included in Appendix.

'REGROUPS' algorithm for grouping ATs into coherent wave fronts were found to require extensive manual review and correction. Spatial analysis showed slow wave propagation to be dynamic, with propagation occurring in the antegrade and retrograde directions (Figs. 3A and 3B). Collisions were observed between slow waves propagating in opposite directions (Fig. 3C). Propagation patterns were dynamic in time, with observed changes from antegrade to retrograde propagation within the duration of the recording periods. Of the 24 total recordings analyzed, 8 (33\%) exhibited solely antegrade propagation across the entire array, $3(13 \%)$ exhibited solely retrograde propagation, and $13(54 \%)$ exhibited both antegrade and retrograde propagation (e.g., colliding wavefronts).

\section{Analysis of Slow Wave Characteristics}

The frequency, amplitude, and velocity of slow waves all decreased along the length of the intestine from proximal duodenum to terminal ileum, but the pattern of decrease varied between each characteristic, as shown in Figure 6. Slow wave frequency gradually decreased in a linear fashion from proximal duodenum to terminal ileum. Slow wave amplitude decreased very rapidly over the short segment of intestine from the proximal duodenum to the proximal jejunum (approximately $0.5 \mathrm{~m}$ ), and then stabilized from there to the terminal ileum. Slow wave velocity decreased from proximal duodenum to mid-jejunum, and then remained relatively stable from there to the terminal ileum. No statistical differences were observed between the frequency, amplitude or velocity of slow waves propagating in the antegrade versus retrograde direction, so results are reported independent of propagation direction. These frequency, amplitude and velocity trends are similar to those documented in other species, ${ }^{3,6,23}$ further validating the analysis pipeline developed in this paper.

\section{Discussion}

This study presents a platform for obtaining reliable small intestine slow wave recordings using mass-produced flexible arrays and computational algorithms for automated slow wave analysis. Several new or improved approaches to small intestine mapping were developed, notably: (1) the flexible electrode arrays allowed for mapping around the intestinal circumference and greater than 2.5 times the length of intestine mapped in previous studies, 6,22 (2) the effect of digital filtering methods were quantified, (3) automated algorithms for small intestine slow wave AT marking were optimized and (4) the data analysis and visualization process was largely automated, including the calculation of slow wave frequency, velocity and amplitude, and the generation of AT, velocity field and amplitude maps. In combination, these outcomes constitute an improved platform for small intestine HR mapping that will allow more thorough serosal mapping, as well as substantial efficiency gains in the data analysis process, while also providing enhanced opportunities for clinical application.

The findings of this study match the trends of decreasing frequency, amplitude, and velocity along the intestine from historical data of other species, particularly the canine (which is the primary model for historic in vivo large animal studies), ${ }^{3,6,23}$ providing experimental validation for the more comprehensive methods developed by this study. However, some quantitative differences 
exist between the porcine and canine slow waves. The porcine small intestine was much longer than that of the canine (approximately $15.5 \mathrm{~m}$ porcine versus approximately $3 \mathrm{~m}$ canine $^{3,6}$ ), potentially contributing to some of the interspecies differences in quantitative slow wave results. The decrease in slow wave frequency along the porcine intestine was similar to that described in several previous canine studies. ${ }^{3,6,23}$ Although the duodenal frequencies were similar, the porcine terminal ileal frequency was less than that of the canine $(8.4 \mathrm{cpm}$ porcine versus $12.2 \mathrm{cpm}$ canine $\left.^{6}\right)$, potentially resulting from the longer porcine intestinal length. Porcine duodenal velocity was much lower than that of the canine $(14.9 \mathrm{~mm} / \mathrm{sec}$ porcine versus $105 \mathrm{~mm} / \mathrm{sec} \mathrm{ca-}$ nine $\left.^{6}\right)$, although velocity in the terminal ileum was similar $(8.8$ $\mathrm{mm} / \mathrm{sec}$ porcine versus $8 \mathrm{~mm} / \mathrm{sec}$ canine $^{6}$ ). Slow wave amplitude is affected by the size and material of the recording electrode, making comparisons between studies using different recording methods difficult and unreliable.

Propagation pattern was more variable in the porcine intestine when compared to a previous HR canine mapping study; ${ }^{6}$ $54 \%$ of porcine recordings displayed both antegrade and retrograde propagation (e.g., colliding wavefronts), whereas only $2 \%$ of the canine recordings displayed such activity, termed 'propagation blocks' in that study. ${ }^{6}$ This increase in wavefront collisions could be due to the increased length of the electrode array used in this porcine study (124 versus $46 \mathrm{~mm}$ in the canine study ${ }^{6}$ ), increasing the likelihood that a collision site would be located within the mapped area. However, the increase in multiple propagation directions may also be indicative of dysrhythmic slow wave activity, which can occur under these experimental conditions in weaner pigs, ${ }^{24}$ further validating the ability of the described methods to map complex propagation patterns.

The techniques applied in this study can readily be translated to human studies, because PCBs can be sterilized and used intra-operatively. ${ }^{7,8}$ Unlike in the stomach, where non-invasive cutaneous electrogastrography provides a basic method of evaluating gastric slow wave abnormalities, ${ }^{25}$ cutaneous intestinal analysis yields signals of very low signal-to-noise ratio that are difficult to interpret and are rarely used. ${ }^{26}$ Consequently, there is a critical lack of studies on human small intestine slow wave patterns, especially relating to the pathology of intestinal motility disorders, which remain a considerable clinical challenge. ${ }^{27}$ The clinical drive for an improved understanding of small intestine slow wave events has recently been reinvigorated by a growing understanding of ICC pathologies. For example, the mutation of the $\mathrm{Na}_{\mathrm{v}} 1.5$ sodium channel has recently been associated with irritable bowel syndrome. ${ }^{10}$ The methods described in this study also create a means to investigate the bioelectrical implications of motility disorders like diabetic dysmotility, ${ }^{9}$ chronic idiopathic pseudo-obstruction, ${ }^{27}$ and mitochondrial neurogastrointestinal encephalomyopathy. ${ }^{28}$

The recordings obtained and analyzed in this study required invasive techniques, as will further investigations using similar techniques in the near future. However, the placement of electrodes directly on the serosa offers the opportunity to map slow waves in the highest detail in normal and diseased human intestine. ${ }^{7,8}$ Once abnormal clinical patterns are defined using a HR electrode array as described in this study, a less-invasive device with fewer channels could potentially be employed to detect dysrhythmia based on signatures of the dysrhythmic patterns, such as changes in amplitude and velocity of extracellular signals. ${ }^{29}$ In the future, once dysrhythmias are better defined by methods like those described in this study, much smaller devices can be utilized and inserted endoscopically, as have already begun to be developed. ${ }^{30}$ While much of the intestine may be unreachable by endoscope, the duodenum is easily accessible endoscopically, and duodenal electrical abnormalities have been implicated in motility disorders. ${ }^{28}$

The small intestine is topologically a flexible cylinder held by the mesentery. It decreases in circumference along its length and is typically collapsed in the fasted state. These attributes, combined with the biological variability between animals, make it challenging to achieve good electrode contact between a HR electrode array and the intestinal serosa, especially in vivo. The $75 \pm$ 9\% contact achieved in this study is a reflection of the 3-dimensionally complex organ geometry (effectively a tube that is held into curved 'loops' by the mesentery), but this electrode contact proved sufficient for spatial analysis of complex and variable slow wave propagation patterns. Importantly, the flexibility of the electrode arrays allowed mapping around the intestinal circumference for the first time and covered approximately $80 \%$ of the circumference in the jejunum.

Appropriate filter settings are vital for the analysis of gastrointestinal slow wave signals, ${ }^{19}$ and the comparison of digital filters performed in this study is a valuable analysis. The specified Savitzky-Golay filter was determined to be the preferred filtering method because it maintained the slow wave amplitude and gradient, which were deemed the most important factors, as only mild noise correction is generally necessary and maintaining signal quality is vital. The Butterworth $120 \mathrm{cpm}$ filter performed similar to the Savitzky-Golay, but the Savitzky-Golay is a time 
domain based filter that better maintains the slow wave peaks, as demonstrated by the amplitude maintenance. ${ }^{31}$

Intestinal HR mapping yields a vast data set, creating a substantial data analysis throughput problem. Slow wave detection, analysis, and visualization have previously been performed manually in the small intestine, ${ }^{5,6}$ presenting a time-intensive barrier to experimental and translational applications. An automated algorithm for marking slow wave activity in conscious animals has previously been presented by Lammers et al, ${ }^{32}$ and is very similar to the amplitude-sensitive differentiator approach tested in this study. The application and tuning of the FEVT algorithm presented in this study constitutes an improvement over this previous approach, ${ }^{12}$ and the addition of automated frequency, amplitude, velocity and visualization techniques yields an invaluable pipeline of analysis tools.

The FEVT parameters summarized in Table 1 represent the set of parameters that yielded the best combined performance across all 3 validation data sets, although they were not the optimal parameter combination for any of the individual data sets. Achieving a PPV and Sensitivity of $90 \%$ and $87 \%$, respectively represents a functional automated detection algorithm that is sufficiently accurate, although manual review will still be necessary when higher reliability is desired.

Regenerative actions potentials, termed 'spikes,' are also known to occur in the small intestine and are closely related to contractile response. ${ }^{1}$ Spikes usually occur during the plateau phase of the slow wave, but are also known to occur in the absence of slow waves, likely modulated by neural inputs, and resulting in propagating contractions. ${ }^{1,15}$ Spikes can propagate independent of slow waves, and are characterized by different velocity and propagation patterns than the corresponding slow wave activity. ${ }^{22,33}$ Although it was not a focus of the current study, the experimental methods developed here could be adapted to map spike activity in the future, and a new automated method for spike detection is being developed.

The recordings in this study were performed on fasted, anesthetized pigs, shortly after laparotomy. The effects of anesthesia on slow wave activity have not been extensively studied. However, previous mapping studies have been successfully performed under anesthesia in several species. ${ }^{6,818}$ To mitigate adverse effects in this study, intestinal handling was kept to a minimum, and temperature and moisture levels were controlled.

In the future, automating the process of detecting and deleting the channels that do not contain slow wave signal content, potentially through frequency or kurtosis analysis, ${ }^{34}$ would further streamline the data analysis pipeline and eliminate the need for these channels to be manually screened. Additionally, automated clustering of slow wave events from individual electrode channels into corresponding wavefronts requires improvement. Although the 'REGROUPS' algorithm was utilized in this study and performed adequately on selected recordings of simple propagation, ${ }^{13}$ extensive manual correction was required when propagation was complex.

In conclusion, the methods developed in this study allow for improved and efficient in vivo studies of small intestine slow wave activity. They represent an overall improved pipeline of experimental recording to data analysis to visualization. Importantly, the methods are applicable for use in human studies. Future studies will now apply these methods to better define organ-level mechanisms of normal and aberrant small intestine slow wave activity, including in human subjects, aiming to improve the clinical understanding of small intestine motility in health and disease.

\section{Acknowledgements}

With great sadness, AJP passed away during the course of this project. We thank Linley Nisbet and Rita Yassi for expert technical assistance. We thank Wenlian Qiao for her contribution to the design of the electrodes.

\section{References}

1. Huizinga JD, Lammers WJ. Gut peristalsis is coordinated by a multitude of cooperating mechanisms. Am J Physiol Gastrointest Liver Physiol 2009;296:G1-G8.

2. Suzuki N, Prosser CL, DeVos W. Waxing and waning of slow waves in intestinal musculature. Am J Physiol 1986(1 Pt 1);250: G28-G34.

3. Szurszewski JH, Elveback LR, Code CF. Configuration and frequency gradient of electric slow wave over canine small bowel. Am J Physiol 1970;218:1468-1473.

4. Du P, O'Grady G, Egbuji JU, et al. High-resolution mapping of in vivo gastrointestinal slow wave activity using flexible printed circuit board electrodes: methodology and validation. Ann Biomed Eng 2009;37:839-846.

5. Lammers WJ, al-Kais A, Singh S, Arafat K, el-Sharkawy TY. Multielectrode mapping of slow-wave activity in the isolated rabbit duodenum. J Appl Physiol 1993;74:1454-1461.

6. Lammers WJ, Ver Donck L, Schuurkes JA, Stephen B. Peripheral pacemakers and patterns of slow wave propagation in the canine small intestine in vivo. Can J Physiol Pharmacol 2005;83:1031-1043.

7. O'Grady G, Angeli TR, Du P, et al. Abnormal initiation and conduction of slow wave activity in gastroparesis defined by high-resolution electrical mapping. Gastroenterology 2012;143:589-598.e1- 
e3.

8. O'Grady G, Du P, Cheng LK, et al. Origin and propagation of human gastric slow wave activity defined by high-resolution mapping. Am J Physiol Gastrointest Liver Physiol 2010;299:G585-G592.

9. Lammers WJ, Al-Bloushi HM, Al-Eisaei SA, et al. Slow wave propagation and plasticity of interstitial cells of Cajal in the small intestine of diabetic rats. Exp Physiol 2011;96:1039-1048.

10. Saito YA, Strege PR, Tester DJ, et al. Sodium channel mutation in irritable bowel syndrome: evidence for an ion channelopathy. Am J Physiol Gastrointest Liver Physiol 2009;296:G211-G218.

11. Morrison P, Miedema BW, Kohler L, Kelly KA. Electrical dysrhythmias in the Roux jejunal limb: cause and treatment. Am J Surg 1990;160:252-256

12. Erickson JC, O'Grady G, Du P, et al. Falling-edge, variable threshold (FEVT) method for the automated detection of gastric slow wave events in serosal high-resolution electrical recordings. Ann Biomed Eng 2010;38:1511-1529.

13. Erickson JC, O'Grady G, Du P, Egbuji JU, Pullan AJ, Cheng LK. Automated cycle partitioning and visualization of high-resolution activation time maps. Ann Biomed Eng 2011;39:469-483.

14. Paskaranandavadivel N, O'Grady G, Du P, Pullan AJ, Cheng LK. An improved method for the estimation and visualization of velocity fields from gastric high-resolution electrical mapping. IEEE Trans on Biomed Eng 2012;59:882-889.

15. Lammers WJ, Stephen B, Slack JR. Similarities and differences in the propagation of slow waves and peristaltic waves. Am J Physiol Gastrointest Liver Physiol 2002;283:G778-G786.

16. Angeli TR, O'Grady G, Erickson JC, et al. Mapping small intestine bioelectrical activity using high-resolution printed-circuit-board electrodes. Conf Proc IEEE Eng Med Biol Soc 2011;2011:49514954.

17. Bayly PV, Johnson EE, Idriss SF, Ideker RE, Smith WM. Efficient electrode spacing for examining spatial organization during ventricular fibrillation. IEEE Trans Biomed Eng 1993;40:1060-1066.

18. Egbuji JU, O'Grady G, Du P, et al. Origin, propagation and regional characteristics of porcine gastric slow wave activity determined by high-resolution mapping. Neurogastroenterol Motil 2010;22:e292e300.

19. Paskaranandavadivel N, Cheng LK, Du P, O'Grady G, Pullan AJ. Improved signal processing techniques for the analysis of high resolution serosal slow wave activity in the stomach. Conf Proc IEEE Eng Med Biol Soc 2011;2011:1737-1740.

20. Yassi R, O'Grady G, Paskaranandavadivel N, et al. The Gastrointestinal Electrical Mapping Suite (GEMS): software for analyzing and visualizing high-resolution (multi-electrode) recordings in spatiotemporal detail. BMC Gastroenterol 2012;12:60.

21. Maragos P, Potamianos A. Higher order differential energy operators. IEEE Signal Process Lett 1995;2:152-154.

22. Lammers WJ, Stephen B, Slack JR, Dhanasekaran S. Anisotropic propagation in the small intestine. Neurogastroenterol Motil 2002; $14: 357-364$.

23. Diamant NE, Bortoff A. Nature of the intestinal slow-wave frequency gradient. Am J Physiol 1969;216:301-307.

24. O'Grady G, Egbuji JU, Du P, et al. High-resolution spatial analysis of slow wave initiation and conduction in porcine gastric dysphythmia. Neurogastroenterol Motil 2011;23:e345-e355.

25. Chen JD, Lin Z, Yin Y. Electrogastrography. In: Parkman HP, McCallum RW, Rao SC, eds. GI motility testing: A laboratory and office handbook. Thorofare, NJ: SLACK Incorporated 2011:81-92.

26. Chen JD, Schirmer BD, McCallum RW. Measurement of electrical activity of the human small intestine using surface electrodes. IEEE Trans Biomed Eng 1993;40:598-602.

27. Jones MP, Wessinger S. Small intestinal motility. Curr Opin Gastroenterol 2006;22:111-116.

28. Scheffer RC, Smout AJ. Tachyduodenia in mitochondrial neurogastrointestinal encephalomyopathy. Neurogastroenterol Motil 2011; 23:408-410.

29. O'Grady G, Du P, Paskaranandavadivel N, et al. Rapid high-amplitude circumferential slow wave propagation during normal gastric pacemaking and dysrhythmias. Neurogastroenterol Motil 2012;24: e299-e312.

30. Farajidavar A, O'Grady G, Rao SM, Cheng LK, Abell T, Chiao JC. A miniature bidirectional telemetry system for in vivo gastric slow wave recordings. Physiol Meas 2012;33:N29-N37.

31. Hargittai S. Savitzky-Golay least squares polynomial filters in ECG signal processing. Comput Cardiol 2005;32:763-766.

32. Lammers WJ, Michiels B, Voeten J, Ver Donck L, Schuurkes JA. Mapping slow waves and spikes in chronically instrumented conscious dogs: automated on-line electrogram analysis. Med Bio Eng Comput 2008;46:121-129.

33. Lammers WJ, Ver Donck L, Schuurkes JA, Stephen B. Longitudinal and circumferential spike patches in the canine small intestine in vivo. Am J Physiol Gastrointest Liver Physiol 2003;285:G1014G1027.

34. Bull S, O'Grady G, Cheng LK, Pullan AJ. A framework for the online analysis of multi-electrode gastric slow wave recordings. Conf Proc IEEE Eng Med Biol Soc 2011;2011:1741-1744. 
Appendix. Average Slow Wave Characteristics Along Intestine

\begin{tabular}{lcccc}
\hline \multicolumn{1}{c}{ Location } & Distance from pylorus $(\mathrm{m})$ & Frequency $(\mathrm{cpm})$ & Amplitude $(\mu \mathrm{V})$ & Velocity $(\mathrm{mm} / \mathrm{sec})$ \\
\hline Proximal duodenum & 0.1 & $16.6 \pm 0.4$ & $145 \pm 22$ & $14.9 \pm 1.2$ \\
Proximal jejunum & 0.5 & $16.4 \pm 0.3$ & $43 \pm 6$ & $13.4 \pm 1.7$ \\
Mid-jejunum & 4.5 & $13.8 \pm 0.5$ & $32 \pm 6$ & $8.8 \pm 0.6$ \\
Proximal ileum & 9.5 & $11.6 \pm 0.8$ & $29 \pm 2$ & $7.6 \pm 0.3$ \\
Terminal ileum & 15.5 & $8.4 \pm 0.7$ & $42 \pm 9$ & $8.8 \pm 0.7$ \\
\hline
\end{tabular}

Frequency, amplitude and velocity at 5 discrete locations along the intestine. Frequency, amplitude and velocity all decreased along the length of the intestine, in accord with the trends shown in Figure 6. 